\title{
Desafios a melhores resultados em alfabetização
}

\author{
Challenges to have better results in literacy
}

Changements pour avoir les meilleurs résultats en alphabétisation

\author{
Leonor SCLIAR-CABRAL
}

\section{RESUMO}

Neste artigo, temos algumas estatísticas de países desenvolvidos, como os EUA e o Reino Unido, bem como o Brasil, com altas taxas de analfabetismo funcional, que são apresentadas. O programa de Iniciativa de Intervenção Precoce inspira outros países a aplicarem medidas similares tais como reformulação dos currículos, das práticas escolares, do material pedagógico; avaliação periódica e recuperação dos alunos que apresentam dificuldades de aprendizagem da leitura e escrita. Será dada ênfase à realidade psicológica da invariância dos traços que diferenciam as letras demonstrada por vários experimentos rodados pela neurociência.

Palavras-chave: analfabetismo funcional, Programa de Iniciativa e Intervenção (EII), consciência fonológica.

\section{ABSTRACT}

In-this article, some statistics related to developed countries, like USA and UK and also Brazil, with high figures of functional illiteracy are presented. The program Early Intervention Initiative (EII) inspires other countries for applying similar measures like the reformulation of curricula, of activities in the classroom, of pedagogical material, of assessment and remediation of students who have difficulties on learning reading and writing. Emphasis will be given to the psychological reality of letters features invariance demonstrated by various experiments which had been recently run by neuroscientists.

Index terms: functional illiteracy, EII, phonological awareness. 


\section{RÉSUMÉ}

Dans cet article, nous avons quelques statistiques de pays développés, comme les E.U.A. et le Royaume-Uni, ainsi que le Brésil, avec de hauts taux d'analphabétisme fonctionnel, qui sont présentés. L'EII inspire autres pays à appliquer des mesures semblables tels comme reformulation des curriculum vitae, des pratiques scolaires, du matériel pédagogique; l'évaluation périodique et la récupération des élèves qui présentent des difficultés d'apprentissage de la lecture et de l'écrite. Sera donné accent à la réalité psychologique de l'invariance des traces qui différencient les lettres démontrée par plusieurs expériences tournoyées par la neuroscience.

Mots clés: analphabétisme fonctionnel, EII, conscience phonologique.

\section{Transformações profundas}

Profundas transformações nas comunicações linguísticas têm sido observadas nos últimos 25 anos, cujo cenário é a globalização acelerada e $\oint$ contato não presencial entre os interlocutores pela Internet: a fusã̉o, do áudio, vídeo e telefonia num só sistema, o uso maciço do computador e a nanotecnologia são exemplos da revolução científica e tecnológica que acarreta relações de trabalho determinadas por quem detém mais conhecimento e está à frente na inovação científica e tecnológica: isto impõe novos processos na formação dos professores, implicando a reformulação dos currículos, dos conteúdos das disciplinas e da própria metodologia de ensino.

Pode parecer que o acesso ao conhecimento foi democratizado e que mais pessoas o dominam no mundo inteiro, rompendo assim o fosso que separa os poderosos dos destituídos, mas isto não corresponde aos fatos: não só ainda existem 774 milhões de iletrados, dos quais 64\% são mulheres, 
conforme os dados do Serviço de Estatística da UNESCO (cf. UNESCO INSTITUTE FOR STATISTICS, 2007), como mesmo em muitos países em que o ensino fundamental é compulsório, a porcentagem de analfabetos funcionais vem aumentando, o que os torna praticamente à margem da realização pessoal, social e civil.

Por ser na minha visão o problema central no contexto do ensinoaprendizagem, focalizarei o analfabetismo funcional.

\section{Alfabetismo funcional}

Dentre as muitas definições de analfabeto funcional, para as quais não há consenso, adotamos a de Scliar-Cabral (SCLIAR-CABRAL, 2003): "O conceito de analfabeto funcional, como o próprio adjetivo indica, deve, contudo, repousar sobre a falta de competência do indivíduo para ler e escrever os textos dos quais necessita em sua vida cotidiana familiar, social e de trabalho". A definição condiz com a da UNESCO (UNESCO INSTITUTE FOR STATISTICS, 2007) de letrado funcional: "É funcionalmente letrada a pessoa que puder engajar-se em todas as atividades nas quais o letramento for condição para o desempenho efetivo no seu grupo e comunidade e também para permitir-lhe que continue a utilizar a leitura, a escrita e $\mathrm{o}^{-}$-álculo para o-seu próprio desenvolvimento e o de sua comunidade."

A partir da própria definição do Programa de Avaliação Internacional dos Alunos (PISA) de que "na leitura, ser letrado é entender, usar e refletir sobre textos escritos, a fim de alcançar as próprias metas para desenvolver o conhecimento e as potencialidades e participar na sociedade" (OCDE, 2003, trad. da autora), as cifras dos analfabetos funcionais são alarmantes, mesmo em países desenvolvidos como os Estados Unidos e o Reino Unido (cabe assinalar que, nestes países a incidência maior é entre os imigrantes, inclusive entre os de segunda geração). 
Uma classificação mais operacional em cinco níveis é a utilizada pelo Literacy Assessment and Monitoring Programme (LAMP), programa de testagem e monitoria da UNESCO (UNESCO INSTITUTE FOR STATISTICS, 2007):

Nível 1: O indivíduo possui habilidades muito pobres e pode nem ser capaz de determinar a dose correta do remédio para dar ao filho, a partir do rótulo da embalagem.

Nível 2: Os entrevistados só conseguem operar tarefas e material escrito simples, disposto com clareza. Conseguem ler, mas se saem mal nos testes de compreensão. Podem ter desenvolvido habilidades de cópia para dar conta das demandas de escrita mais corriqueiras, mas acham difícil enfrentar novos desafios como os exigidos no trabalho.

Nível 3: Considerado o mínimo adequado para dar conta das demandas diárias e de trabalho, numa sociedade complexa. É o nível em geral requerido para completar a escola secundária e entrar na universidade.

Níveis 4 e 5: Os entrevistados demonstram domínio das habilidades para processar informação mais complexa.

\section{Dados alarmantes}

O Departamento de Edueação do Reino Unido em seu relatório de 2006 informou que a cada ano, 100.000 alunos deixam a escola como analfabetos funcionais no Reino Unido (cf. GUARDIAN UNLIMITED, 10/07/2007). Embora a taxa de letramento, nos Estados Unidos seja muito elevada, mensurada por no mínimo oito anos de escolaridade, estatísticas recentes indicam a existência de aproximadamente 30 milhões de analfabetos funcionais, cifra que vem aumentando (CIVILLIBERTIES, 2007). Observe-se, portanto, que frequentar a escola mesmo até completar o ensino fundamental não é garantia para que o indivíduo consiga entender, usar e refletir sobre os textos escritos. Somente as instituições que 
investiram pesado na formação do magistério e adotaram métodos e materiais advindos das pesquisas avançadas conseguiram resultados satisfatórios no domínio da leitura e escrita por parte da população, estando em primeiro lugar dentre os 31 países associados à OCDE (OCDE, 2005) a Finlândia, com a melhor média: o México, único país latino-americano associado, ocupou a pior posição, com (404,9 pontos). No Brasil, a situação do analfabetismo funcional continua grave: segundo os resultados da quinta edição da pesquisa do Indicador de Analfabetismo Funcional (INAF), "somente $26 \%$ dos brasileiros que têm entre 15 e 64 anos dominam plenamente a leitura e a escrita no Brasil" (cf. INAF, 2007). Conforme Cipolla (2010), "74\% da população brasileira não consegue entender um texto simples (segundo o Inaf). Apenas $25 \%$ da população brasileira adulta é plenamente alfabetizada. O dado é do Inaf 2009. Apenas $9,8 \%$ dos alunos do $3^{\circ}$ ano do ensino médio sabem o conteúdo esperado em Matemática e 24,5\%, o de Língua Portuguesa. Fonte: Todos Pela Educação, com base nos dados do Sistema de Avaliação da Educação Básica (Saeb) 2007. 74\% da população brasileira não consegue entender um texto simples (cf. INAF)".

Cabe investigar as causas de tal situação, para indagar como a Psicolinguística Aplicada poderá contribuir para reverter o quadro.

Uma das possibilidades é conhecer quais as medidas que foram tomadas em cenários que apresentavam taxas alarmantes de analfabetismo funcional e que conseguiram baixá-las consideravelmente; outra é aplicar os mais recentes achados da Neurociência sobre a aprendizagem da leitura, a fim de entender quais as maiores dificuldades a serem vencidas em tal aprendizagem.

\section{O Programa Iniciativa de Intervenção Precoce}

Tomaremos como exemplo o programa Inciativa de Intervenção Precoce (Early Intervention Initiative (EII)), desenvolvido pelo Conselho do 
Condado Oeste de Dunbartonshire, na Escócia e que, em junho último, recebeu o prestigioso prêmio do Municipal Journal pela maior conquista em assistência à criança no Reino Unido (WEST DUNBATONSHIRE COUNCIL, 2007).

O programa começou em 1997, com a meta para ser atingida em dez anos. Em 1997, somente 5\% das crianças que frequentavam a primeira série do primário conseguiam escores altos em leitura: com a aplicação do programa, a cifra subiu para $45 \%$. A reversão do problema também se pode observar no fato de que em 1997, as crianças com escores baixos que frequentavam a segunda série do primário, constituíam 11\% e em 2007 baixaram para $1 \%$.

Sem que possamos entrar em detalhes, por limitações de tempo, o programa prioriza a educação infantil, desenvolvendo a consciệncia fonológica na pré-escola e utilizando basicamente o método fônico sintético e o enfoque multissensorial, com material pedagógico elaborado a partir de pesquisas (Jolly Phonics); atividades de intervenção, com uma equipe de professores especialmente treinados; avaliação e monitoria contínuas; tempo extra para a leitura no currículo, assessoria às famílias e de quem cuida das crianças e a implementação de um entorno de letramento na comunidade (Education Guardian, 2007).

É preciso enfatizar a vontade política da administração do condado que investiu no projeto de erradicar o analfabetismo funcional em dez anos, mais precisamente, no mês de novembro de 2007, inclusive convocando especialistas como assessores: o projeto considerou importante o concurso da família e da comunidade e foi desenvolvido um trabalho para suplementar o chamado currículo escondido. A efetividade de tais medidas para erradicar o analfabetismo funcional no Reino Unido vem confirmada pelo comentário da Chefe dos Inspetores de sua Majestade, Christine Gilbert (GILBERT, 2007, p. 2) ao Relatório Anual de 2006/07. Também recomendou que o trabalho fônico de alta qualidade fosse o enfoque 
privilegiado para ensinar as crianças a ler, de modo que pudessem se deslocar mais suavemente de "aprender para ler", para "ler para aprender".

\section{Reflexões sobre o Programa Iniciativa de Intervenção Precoce}

Dado o fato de que muitos projetos para implementar o letramento apresentaram resultados tão insatisfatórios, uma das primeiras tendências é copiar, sem reflexão ou adaptação a cenários diferentes, o que deu resultados noutros lugares.

É evidente que algumas medidas são indiscutíveis, por exemplo, a vontade política das instituições responsáveis pela educação de convocâr os especialistas em ensino-aprendizagem da leitura e escrita para assessorarem em larga escala os educadores do ensino pré-escolar e fundamentạl, bem como os autores do respectivo material pedagógico. Isso implica a presença não só de pedagogos e psicólogos altamente especializados, como ò concurso de linguistas, psicolinguistas e fonoaudiólogos, que deverão se ocupar da reformulação dos currículos, da formação contínua do magistério, das práticas escolares, do material pedagógico, da avaliação periódica e da recuperação dos alunos que apresentam dificuldades de aprendizagem da leitura e escrita.

A compreensão por parte dos professores das bases científicas que fundamentam, por exemplo, a base fônica, impedirá a prática mecânica e inadequada dos exercícios, o que redundaria no efeito inverso ao desejado.

Com efeito, a razão primordial que fundamenta a fônica é que a base dos sistemas alfabéticos, ou seja, os grafemas (formados por uma ou mais letras) representam um fonema (classe de sons com função de distinguir significados). Ora, isto vai contra a percepção que o indivíduo tem da fala antes de se alfabetizar, ou seja, a fala é percebida como um contínuo, residindo, aí, a maior dificuldade na aprendizagem da leitura e da escrita. 
Portanto, um trabalho sistemático tem que ser desenvolvido para que o indivíduo reconstrua de modo consciente a percepção da fala e possa desmembrar a sílaba em seus constituintes.

Enfatizamos, porém, que tanto os fonemas quanto sua representação, os grafemas, têm a função de distinguir significados. Ignorar que tanto a língua oral quanto a escrita fazem parte dos sistemas semióticos é negar que servem para comunicar pensamentos.

Outro risco decorrente da falta de conhecimentos de fonética para quem ensina fônica é pensar que é possível ouvir ou articular isoladamente uma consoante menos continua, isto é, uma oclusiva.

Outra questão teórica é desconhecer que a decodificação precisal ser aprendida pelos valores que as letras têm, muitas vezes condicionadas pelo contexto, e não por seus nomes: evidentemente, a palavra "bola" não se lê como "beóélia".

Finalmente, gostaria de mencionar que, além das questões que remontam à educação pré-escolar e fundamental e ao preparo dos professores, a explosão lexical e semântica e de universos especializadoß ocasionada pelas revoluções científicas e tecnológicas aceleradas, determinou a formação de verdadeiros guetos linguísticos, impermeáveis mesmo para os considerados bons leitores. Isto vem determinando o fracionamento do conhecimento e a impossibilidade de compreender os inúmeros universos cognitivos: o ideal humanístico se encontra cada vez mais distante.

\section{Contribuições da Neurociência para a alfabetização}

Experimentos realizados mais recentemente pela neurociência demonstram a realidade psicológica das invariâncias dos traços que constituem as letras. Com efeito, as evidências obtidas por Polk e Farah 
(2002) favorecem a hipótese de uma área que processa a forma abstrata, não perceptual da palavra.

Uma das razões para o grande interesse que tais descobertas suscitam reside no fato de esclarecerem quais as dificuldades com as quais se defrontam os aprendizes de um sistema escrito, particularmente nos sistemas alfabéticos, e como contorná-las.

Nos mamíferos, os neurônios são programados para processar o sinal visual simetricamente, pois, para a sobrevivência, é economicamente mais eficiente, para o reconhecimento dos objetos, descartar as diferenças que eventualmente possam existir nos sinais entre a direção para a esquerda ou para a direita, para cima ou para baixo e, assim, eles são reconhecidos cỏmo simétricos. Mas não é isto o que ocorre no reconhecimento das letras: os neurônios precisam aprender como reconhecer tais diferenças, que são absolutamente pertinentes para aprender a ler. A necessidade é dramática no caso das letras em espelho, como b/d, p/q, b/p, d/q, n/u, a/e. Graçàs à plasticidade neuronal, os neurônios podem ser reciclados (cf. ASSAF; BASSER, 2004; BARLASSOV; WEINSTEIN; HOCHSTEIN, 2004; CHANGEUX, 2007, p. 18; DEHAENE, 2007, p. 27; HAREL et al., 2004; SPERBER, 1996), se o contexto de ensino-aprendizagem for adequado, pois, do contrário, os alunos continuarão a apresentar suas dificuldades iniciais, sendo, com muita frequência rotulados como disléxicos.

As neurociências usam três técnicas principais na situação experimental: a Imagem por Ressonância Magnética (IRM), a electroencefalografia (EEG), e, o mais eficiente método para medir o processamento da leitura, a magneto electroencefalografia (MEG).

Algumas evidências empíricas obtidas pelos experimentos confirmam princípios já conhecidos como os de que as regiões primárias processam os sinais brutos, independentemente da especialização hemisférica, no caso do sinal luminoso, a região occipital central. 
O primeiro processamento leva aproximadamente 100 milissegundos. Embora haja alguns pontos controversos na literatura, faltando ainda esclarecer (cf. STARR; RAYNER, 2001) a discrepância entre o baixo nível dos fatores oculares se comparado ao alto nível dos processos cognitivos para afetarem os movimentos oculares, ou quanta informação é extraída à direita do olho, ou, ainda, se a fóvea central pode abarcar mais de uma palavra, há um relativo consenso quanto ao fato de existir uma limitação drástica de captação no momento da fixação. Por isso, os olhos correm pela linha, em movimentos de sacada (quatro ou cinco por segundo), quando não se vê nada, e param num ponto, a fixação: nos sistemas com direção da esquerda para a direita, a fóvea consegue abarcar três ou quatro caracteres à esquerda do centro do olhar, e sete ou oito à direita (cf. McCONKIE; RAYNER, 1975), decompondo os sinais luminosos em miríades de pontos que, metaforicamente, chamo de píxeis e, só depois da recomposição em formas invariantes que possam emparelhar com as dos respectivos neurônios, são enviadas para a área especializada, a região occípito-temporal ventral esquerda.

A novidade consiste em acompanhar on line, como o out-put desse primeiro processamento é enviado compulsoriamente para_a região especializada para a leitura, a região occípito-temporal ventral do hemisfério esquerdo, se o sujeito aprendeu o código escrito, independentemente do sistema (alfabético ou ideográfico). Experimentos conduzidos por Dehaene e colegas (2002) demonstraram que a região occípito-temporal ventral esquerda se ilumina quando os sujeitos são submetidos a palavras escritas, mas não quando as mesmas palavras são ouvidas. É preciso assinalar que a região occípito-temporal ventral esquerda também processa faces, objetos e instrumentos, mas somente algumas partes da região occípito-temporal ventral esquerda preferem o reconhecimento das palavras escritas, enquanto o a região contra-lateral do hemisfério direito prefere o reconhecimento de faces (cf. TARKIAINEN et al., 2002). 
Esses achados confirmam as conclusões de Allinson e colegas (1999), os quais aplicaram a eletroencefalografia a mais de cem pacientes epiléticos enquanto não estavam em crise.

Outra importante evidência empírica obtida pela neurociência diz respeito ao processamento das invariâncias.

O reconhecimento das invariâncias é possível e necessário por duas razões, fundamentalmente: primeira, porque, como mecanismo adaptativo, o sistema visual dos primatas deve reconhecer as formas básicas do que se encontra na natureza, independentemente das variantes que o olhar capta, conforme a distância, o ângulo de visão, a incidência da luz e sombra e a parte em relação ao todo, etc (cf. GRAINGER; REY; DUFAU 2008; SPRATTING 2005); segunda, porque, e essa é especificamente humana, só essa explica a capacidade dos neurônios da região occípito-temporal-ventral esquerda para reconhecer os traços invariantes que compõem as letras: na espécie humana, os respectivos axônios (prolongamentos dos neurônios para levarem a informação a outros neurônios através do mecanismo denominado de sinapse) estão ligados a todas as regiões que processam a linguagem verbal e simultaneamente à região que processa o significado.

A primeira invariância observada no reconhecimento da palavra escrita é_a invariância espacial: seja qual for o sistema de escrita, o seu processamento ocorre obrigatoriamente na região occípito-temporal ventral esquerda.

Nessa região, os neurônios reciclados para o reconhecimento das invariâncias dos traços que compõem as letras, os sintetizam em uma ou duas letras que constituem os grafemas. Como o nome da região indica, há uma associação entre a região occipital ventral esquerda e a região temporal contígua, responsável pelo processamento dos fonemas, mas o processamento em ambas não é simultâneo: o reconhecimento dos grafemas precede sua associação ao respectivo fonema, numa diferença de 
aproximadamente 40 milissegundos, conforme demonstram os experimentos de priming.

$\mathrm{Da}$ associação entre grafemas e fonemas, ambos com a função de distinguir significados, conclui-se pela natureza mais abstrata de tais construtos.

A invariância espacial é comprovada pelos experimentos que demonstram que ler em mandarim ativa a mesma região occípito-temporal ventral esquerda: não são observadas diferenças substanciais entre as características de leitura desses sujeitos e as dos sujeitos que lêem nos sistemas alfabéticos, o que também foi observado quando lêem em kanji ou kana.

Outras evidências sobre a invariância espacial provêm do acompanhamento on line do processamento da palavra escrita. É bem conhecido que as projeções visuais são cruzadas: os sinais luminosos que se apresentam à esquerda se projetam sobre a metade direita da retina de cada olho, de onde a informação é enviada em direção às áreas visuais primárias na região occipital do hemisfério direito (posteriormente enviada via corpus callosum para o hemisfério esquerdo); e os sinais luminosos apresentados à direita se projetam sobre a metade esquerda da retina de cada olho, ambas tratadas na região occípito-temporal ventral do hemisfério esquerdo.

A técnica por IRM demonstra que o processamento unilateral na região V4 dura de 160 a 170 milissegundos. Subitamente, o output converge para a região occípito-temporal ventral esquerda, não importa se os estímulos foram apresentados no lado direito ou esquerdo da tela. Isso é possível porque a conexão entre os dois hemisférios é mediada pelo corpus callosum, consequentemente, se um paciente sofrer uma lesão vascular aí, estará impossibilitado de reconhecer as palavras apresentadas ao lado esquerdo da tela, uma síndrome denominada hemialexia.

Um grande número de experimentos demonstrou um outro tipo de invariância, a invariância de fonte: os neurônios da região occípito-temporal 
ventral esquerda, depois de reciclados, têm a capacidade de reconhecer uma letra como a mesma, apesar de suas múltiplas variantes. Os neurônios da região occípito-temporal-ventral esquerda reconhecem os traços invariantes que compõem as letras, cujos valores são os mesmos, independentemente de seu tamanho, da caixa (MAIÚSCULA ou minúscula), da fonte e estilo (imprensa, manuscrita, itálico, negrito ou sublinhado, etc.), ou da posição que ocupam na palavra (cf. DEHAENE, 2007).

Se o método global fosse correto, o reconhecimento da palavra escrita ocorreria por configuração, exatamente o que o hemisfério direito faz: ele reconhece GALA e gala como palavras distintas (DEHAENE et al., 2004).

$\mathrm{O}$ mesmo se pode dizer em relação à inferência das pessoas em relação a uma criança de três ou quatro anos, quando reconhece na garrafa o rótulo Coca-Cola, de que tal criança já sabe ler. Se a palavra COCA COLA estiver escrita numa folha de papel em letra de imprensa, ou mesmo, se forem escritas outras palavras com as mesmas letras, como CACO ou CALO, obviamente, a criança não as saberá ler, pois reconheceu Coca-Cola por configuração e/ou pelas pistas extra-linguísticas.

Outro achado interessante é o de que os neurônios da região occípito-temporal ventral esquerda além de aprenderem a reconhecer os traços invariantes das letras e suas combinações, também aprendem as regras grafotáticas de uma dada língua. O que são as regras grafotáticas? São as que ditam qual a posição possível, preferencial ou proibida de uma letra. Por exemplo, no português, a letra $\mathbf{h}$ pode ocupar posição inicial antes de vogal; medial somente depois de $\mathbf{c}, \mathbf{l}$ e $\mathbf{n}$ e final somente em interjeições. Os experimentos demonstram que os neurônios da região occípito-temporal ventral esquerda preferem cadeias bem formadas de letras numa dada língua (COHEN et al., 2002).

Tais dados demonstram que tais capacidades não são inatas e precisam ser aprendidas, pois dependem da ortografia de cada língua. 


\section{Considerações finais}

Neste artigo me propus debater qual a prioridade para a formação de professores, optando pelas contribuições ao combate ao analfabetismo em todas suas vertentes, particularmente, ao analfabetismo funcional. Apresentei estatísticas de países desenvolvidos como os EUA e o Reino Unido, e também mencionei o Brasil, com altas taxas de analfabetismo funcional. Mencionei o programa de dez anos, Iniciativa de Intervenção Precoce do West Dunbartonshire Council, cujos bons resultados inspiram outros países a aplicarem medidas similares, mas estando atentos a adaptálas aos respectivos cenários com uma crítica reflexiva, ou seja, indicando as áreas onde é preciso atuar: reformulação dos currículos, das práticas escolares, do material pedagógico; articulação do trinômio escola, família, comunidade; avaliação periódica e recuperação dos alunos que apresentam dificuldades de aprendizagem da leitura e escrita, bem como a formąção contínua do magistério. Finalizei, A seguir, para abordar a questão das invariâncias dos traços que compõem as letras, discorri sobre a programação dos neurônios da visão para simetrizar os sinais. Apresentei as evidências empíricas que comprovam o processamento na região occípiot-temporal ventral esquerda das invariâncias dos traços que compốem as letras, sintetizadas nos grafemas associados aos fonemas, ambos com a função de distinguir significados.

\section{Referências bibliográficas}

ASS AF, Y.; BASSER, P. J.. Virtual histology of human white matter by diffusion MR imaging. Abstracts of the $13^{\text {th }}$ Annual Meeting of Israel Society for Neuroscience. Disponivel em: <http://www.hindawi.com/journals /np /2005/859245.abs.html>. Acesso em 22 ag. 2009.

BARLASSOV, A.; WEINSTEIN, M.; HOCHSTEIN, S. Do odd ilusory shapes pop out? Effects of learning and expertise. Abstracts of the $13^{\text {th }}$ 
Annual Meeting of Israel Society for Neuroscience 2004. Disponivel em: <http://www.hindawi.com/journals/np/ 2005/859245.abs.html>. Acesso em 22 ag. 2009.

CHANGEUX, J.-P. Préface. In: DEHAENE, S.. Les neurones de la lecture. Paris: Odile Jacob, 2007.

CIPOLLA, M. C.. Não somos campeões em Educação. Disponível em: <http://educarparacrescer.abril.com.br/blog/boletimeducacao/2010/06/29/po r-nao-somos-campeoes-em-educacao/>. Acessado em $01 \mathrm{dez} .2010$.

CIVILLIBERTIES. The teleconference about functional illiteracy. Disponivel em: <http://civliber.blogs.bftf.org/2007/07/27/theteleconference-about-functional-illiteracy/>. Acessado em 24 out. 2007.

COHEN, L.; LEHERECY, S.; CHOCHON, F.; LEMER, C.; RIVAUD, S.; DEHAENE, S.. Language-specific tuning of visual cortex? Functional properties of the visual word form area. Brain, 125 (Pt 5), 1054-1069, 2002.

DEHAENE, S.. Les neurones de la lecture. Paris: Odile Jacob, 2007.

Le CLECH; G. POLINE, J. B. et al.. Cerebral mechanisms of word masking and unconscious repetition priming. Nat. Neurosci, 4 (7), ps. 752758, 2002.

GILBERT, C.. Commentary by Her Majesty's Chief Inspector. The Annual Report of Her Majesty's Chief Inspector 2006/07. Disponível em $<\mathrm{http}$ ///live.ofsted.gov.uk/publications/annualreport0607/commentary/page_ 1.htm>. Acessado em 29 de out. 2007.

GRAINGER, J.; REY, A.; DUFAU, S. Letter perception: from pixels to pandemonium. Trends in Cognitive Sciences, 12(10), ps. 381-387, Oct. 2008.

GUARDIAN UNLIMITED. Sounds incredible. The Guardian. Tuesday July $10,2007.2$ Disponível <http://education.guardian.co.uk/egweekly/story/0,,2122125,00.html>. Acessado em 24 out. 2007.

HAREL. A. et al.. Selective neural responses to objects of expertise in experts' ventral visual pathway. Abstracts of the $13^{\text {th }}$ Annual Meeting of Israel Society for Neuroscience 2004. Disponível em <http://www.hindawi.com/journals/np/2005/859245.abs.html>. Acessado em 22 ag. 2009. 
INAF. Encontro nacional reúne instituições que combatem o analfabetismo funcional. Boletim INAF. Disponível em <http://www.ipm.org.br/ipmb_pagina.php?mpg=4.03.00.00.00\&ver=por >. Acessado em 29 out. 2007.

MCCONKIE, G. W.; RAYNER. K.. The span of the effective stimulus during a fixation in reading. Perception and Psychophysics. Percept Psychophys 17, ps. 578-586, 1975.

OCDE. OCDE in figures. Disponível em <http://ocde.p4.siteinternet.com/ publications/ doifiles/012005061T032.xls>. Acessado em 24 out. 2007.

PISA 2003. Country Profiles. Disponível em <http://pisacountry.acer.edu.au/. Acessado em 24 out. 2007.

POLK, T. A.; FARAH, M. J.. Functional MRI evidence for an abstract, not perceptual word-form area. J Exp Psychol Gen., 131(1), ps. 65-72, 2002.

SCLIAR-CABRAL, L.. Revendo a categoria "analfabeto funcional". Revista CrearMundos, no 3 (especial,"Año del libro", 2003). Dispónível em <http://www.wdcweb.info/news/displayarticle.asp?id=12752>. Acessado em 13 out. 2009.

SPERBER, D.. Explaining culture: A naturalistic approach. London: Blackwell, 1996.

STARR, M. S.; RAYNER, K.. Eye movements during reading: sóme current controversies. Trends in Cognitive Sciences, 5(4) 1, ps. 156-163, April 2001.

TARKIAINEN, A.; CORNELISSEN, P. L. \& SALMELIN, R.. Dynamics of visual feature analysis and object-level processing in face versus letterstring perception, Brain, 125 (Pt 5), ps., 1125-1136, 2002.

UNESCO. Institute for Statistics. Literacy survey. 2007. Disponível em <www.uis.unesco.org/profiles/selectCountry_en.aspx.12752>. Acessado em 29 out. 2007.

WEST DUNBARTONSHIRE COUNCIL. Literacy initiative wins major award. News Room. Disponível em <http://www.wdcweb.info/news/displayarticle.asp?id=12752>. Acessado em 29 out. 2007. 


\section{Autora:}

Leonor Scliar-Cabral

Professora da Universidade Federal de Santa Catarina. Pesquisadora do CNPq.

Contato: 1sc@th.com.br

Artigo recebido em junho de 2009.

Artigo aprovado para publicação em agosto de 2009.

\section{Como citar este texto:}

SCLIAR - CABRAL, L.. Desafios a melhores resultados em alfabetização. Revista Acolhendo a Alfabetização nos Países de Língua Portuguesa, Brasil, São Paulo, volume 1, nº. 10, pp. 106 - 122, Mar. 2011. Disponível em: 〈http://www.acoalfaplp.net>. 